\title{
Magnetic Properties of Soft Magnetic FeSi Composite Powder Cores
}

\author{
M. Lauda ${ }^{a, *}$, J. FÜZeR ${ }^{a}$, J. FÜzerová ${ }^{b}$, P. KolláR ${ }^{a}$, M. StreČKováa ${ }^{c}$, M. FÁberovác \\ ${ }^{a}$ Institute of Physics, Faculty of Sciences, P.J. Šafárik University, Park Angelinum 9, 04154 Košice, Slovakia \\ ${ }^{b}$ Faculty of Mechanical Engineering, Technical University, Letná 9, 04200 Košice, Slovakia \\ ${ }^{c}$ Institute of Materials Research SAS, Watsonova 47, 04001 Košice, Slovakia
}

\begin{abstract}
Soft magnetic composites (SMCs), which are used in electromagnetic applications, can be described as ferromagnetic powder particles surrounded by an electrical insulating film. These composite materials offer several advantages over traditional laminated steel cores such as reduction in weight and size. They have some unique properties, including three-dimensional isotropic ferromagnetic behaviour, very low eddy current loss, relatively low total core loss at medium and high frequencies, high electrical resistivity and good relative magnetic permeability. FeSi powder was used as a base ferromagnetic material for preparation of soft magnetic composites. The commercial FeSi particles of a precise spherical shape were prepared in two granulometric fractions (up to $150 \mu \mathrm{m}$ or up to $356 \mu \mathrm{m})$. The phenol-formaldehyde resin modified by $\mathrm{SiO}_{2}$ nanoparticles was used as an electroinsulating layer. The FeSi particles covered by the synthesized resin were compacted at $800 \mathrm{MPa}$ into the ring samples for magnetic measurements. The final samples were treated thermally under the curing schedule, which was suggested according to thermal degradation of the modified resin.
\end{abstract}

DOI: 10.12693/APhysPolA.126.144

PACS: 81.05.Ni, 75.50.Bb, 07.55.-w

\section{Introduction}

Soft magnetic composites offer an interesting alternative to traditional materials such as soft ferrites and electrical steels in applications with alternating magnetic fields. Their structure leads to a significant reduction of the eddy current loss contribution, which become important at high frequencies [1-4]. In this paper we present results on the magnetic losses and complex permeability and their frequency dependence in $\mathrm{FeSi}$ powder in two granulometric fractions. The FeSi particles were covered by the phenol-formaldehyde resin modified by $\mathrm{SiO}_{2}$ nanoparticles and were compacted at $800 \mathrm{MPa}$ into the ring-shaped samples for magnetic measurements.

\section{Experimental}

For preparation of soft magnetic composites was used FeSi powder with two granulometric fractions (up to $150 \mu \mathrm{m}$ or up to $356 \mu \mathrm{m}$ ), prepared by conventional powder metallurgy. The phenol-formaldehyde resin (4 wt.\%), modified by $\mathrm{SiO}_{2}$ nanoparticles, was used as an electroinsulating layer. The FeSi particles were compacted at $800 \mathrm{MPa}$ into the ring-shaped samples and treated thermally under the curing schedule up to $200{ }^{\circ} \mathrm{C}$, which was suggested according to thermal degradation of the resin.

The ring samples were used for magnetic measurements and specific electrical resistivity was measured

\footnotetext{
*corresponding author; e-mail: matej.lauda@post.sk
}

by adapted four-contact method. Complex permeability spectra were measured by an impedance analyser (HP4194A) in the frequency range of $1 \mathrm{kHz}-40 \mathrm{MHz}$. The DC hysteresis loops at maximum induction $0.1 \mathrm{~T}$ were measured by a fluxmeter based hysteresisgraph. The AC hysteresis loops, were measured at frequency range from $50 \mathrm{~Hz}$ to $100 \mathrm{kHz}$, at maximum induction $0.1 \mathrm{~T}$, by two different $\mathrm{AC}$ hysteresisgraphs. In the frequency range from $50 \mathrm{~Hz}$ to $1 \mathrm{kHz}$ by AC/DC Permeameter AMH-1K-S and in the frequency range from $1 \mathrm{kHz}$ to $100 \mathrm{kHz}$ by a MATS-2010SA hysteresisgraph. Total losses were calculated directly from the measured hysteresis loops.

\section{Results}

Figure 1 shows the real and imaginary parts of complex permeability for sample $\mathrm{FeSi}$ in two granulometric fractions. Specific electrical resistivity of the FeSi particles, with particle size up to $150 \mu \mathrm{m}$ is $\rho_{S}=2.0 \Omega \cdot \mathrm{m}$ and $\rho_{S}=0.9 \Omega \cdot \mathrm{m}$ for particle size up to $356 \mu \mathrm{m}$, respectively. Density of particles with size up to $150 \mu \mathrm{m}$ is $\rho=6300 \mathrm{~kg} \cdot \mathrm{m}^{-3}$ and $\rho=6600 \mathrm{~kg} \cdot \mathrm{m}^{-3}$ for particle size up to $356 \mu \mathrm{m}$, respectively. The real part of complex permeability increases with increasing the density of the composite sample [5]. The sample of FeSi particles with higher effective particle size, exhibit the higher values of real part of complex permeability up to $800 \mathrm{kHz}$ (Fig. 1) as a result of higher density of the sample. The imaginary part of complex permeability is linearly proportional with specific electrical resistivity and it is in inverse squared relationship with the particle size [6]. The relaxation and resonance frequency are above upper limit of the impedance bridge. The lower value (about $2 \mathrm{MHz}$ ) of 
this frequency (mostly relaxation processes) for the FeSi sample with particle size up to $356 \mu \mathrm{m}$ is the result of higher particle size and lower electrical resistivity.

The losses in the soft magnetic composites materials can be divided into three types, including the hysteresis, the eddy current and the excess loss. At low frequencies, the hysteresis loss is the main part of total losses. This part of loss strongly depends on particle size, defects, impurities and internal microstrain introduced during compaction. The classical eddy current loss in composite materials can be separated into two parts. One part that originates from the eddy currents, flowing in the entire cross-section of the material, and one part that is due to the eddy currents set up in the interior of a powder particle. The total eddy current loss is a combination of the parts, which are determined by the size, shape and electrical resistivity of the specimen and powders [7].



Fig. 1. Complex permeability spectra (real and imaginary parts) for two composite ring samples FeSi with different granulometric fraction measured at frequency range from $1 \mathrm{kHz}$ to $40 \mathrm{MHz}$.

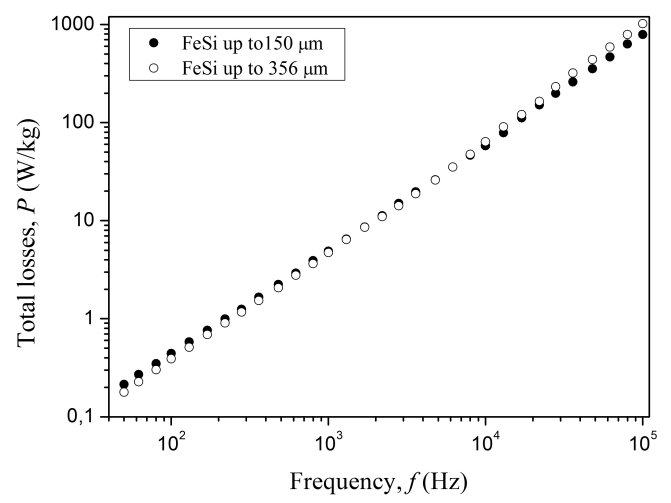

Fig. 2. Total losses $P$ as a function of frequency for two composite ring samples FeSi with different granulometric fraction measured at maximum induction $0.1 \mathrm{~T}$ in the frequency range from $50 \mathrm{~Hz}$ to $100 \mathrm{kHz}$.

The dependences of total losses on the frequency, for the ring samples $\mathrm{FeSi}$ in two granulometric fraction $(150 \mu \mathrm{m}$ and $356 \mu \mathrm{m}$ ), measured in the frequency range from $50 \mathrm{~Hz}$ to $100 \mathrm{kHz}$ at maximum induction $0.1 \mathrm{~T}$, are depicted in Fig. 2.

At low frequencies the hysteresis losses are the major contribution to the total losses, and in composite material are strongly dependent on particle size. Therefore the FeSi sample with higher particle size exhibits lower total losses at low frequencies. When the frequency increases the effect of eddy currents is not negligible. As we can see in Fig. 2 the FeSi sample with lower particle size exhibits lower total losses at high frequencies. At higher frequencies the eddy current losses are significantly lower due to the smaller size of the particles and higher electrical resistivity.

\section{Conclusions}

From the investigation of magnetic properties of two granulometric fractions of FeSi composites materials, it was found that size of the powder particles was responsible for different soft magnetic properties of powder cores. The real part of the permeability is influenced mostly by the density of the composite material. Imaginary $\mu^{\prime \prime}$ part of the complex permeability reflects the power loss due to eddy currents and hysteretic response. Higher total losses at low frequencies in the sample with lower particle size are caused by higher hysteresis losses, which strongly depend on particle size, and at low frequencies these losses are the main part of core loss. The lower total losses at high frequencies in this sample are caused by lower eddy current losses and higher electrical resistivity, which are not negligible at higher frequencies.

\section{Acknowledgments}

This work was realized within the frame of the projects, ITMS 26220120019, ITMS 26220220105, which are supported by the Operational Program financed through European Regional Development Fund. This work was supported by the Slovak Research and Development Agency under the contract No. APVV-0222-10 MAGCOMP and by the Scientific Grant Agency of the Ministry of Education of Slovak Republic and the Slovak Academy of Sciences, projects No. 1/0861/12 and No. 1/0862/12. This work was also supported by the project VVGS-PF2013-106.

\section{References}

[1] A.H. Taghvaei, H. Shokrollahi, K. Janghorban, H. Abiri, Materials and Design 30, 3989 (2009).

[2] A.H. Taghvaei, A. Ebrahimi, K. Gheisari, K. Janghorban, J. Magn. Magn. Mater. 322, 3748 (2010).

[3] P. Kollár, J. Füzer, R. Bureš, M. Fáberová, IEEE Trans. Magn. 46, 467 (2010).

[4] M. Strečková, L. Medvecký, J. Füzer, P. Kollár, R. Bureš, M. Fáberová, Mater. Letters 101, 37 (2013).

[5] H. Shokrollahi, K. Janghorban, F. Mazaleyrat, M. Lo Bue, V. Ji, A. Tcharkhtchi, Materials Chemistry and Physics 114, 588 (2009).

[6] F. Mazaleyrat, L.K. Varga, J. Magn. Magn. Mater. 215-216, 253 (2000).

[7] C. Appino, O. Bottauscio, O. de la Barriere, F. Fiorillo, A. Manzin, C. Ragusa, IEEE Trans. Magn. 48, 3470 (2012). 